\title{
Clinical and radiological determinants of prestroke cognitive decline in a stroke cohort
}

T Pohjasvaara, R Mäntylä, H J Aronen, M Leskelä, O Salonen, M Kaste, T Erkinjuntti

\begin{abstract}
Objectives-Stroke seems to be related to dementia more often than previously assumed and vascular factors are also related to Alzheimer's disease. The pathophysiology of poststroke dementia includes ischaemic changes in the brain, a combination of degenerative and vascular changes, and changes only related to Alzheimer's disease. Some cognitive decline recognised after a stroke may be due to pre-existing cognitive decline. The aim of this study was to determine the clinical and radiological determinants of prestroke cognitive decline.
\end{abstract}

Methods-The study group comprised 337 of 486 consecutive patients aged 55 to 85 years who 3 months after ischaemic stroke completed a comprehensive neuropsychological test battery; structured medical, neurological, and mental status examination; interview of a knowledgeable informant containing structured questions on abnormality in the cognitive functions; assessment of social functions before the index stroke; and MRI.

Results-Frequency of prestroke cognitive decline including that of dementia was $9.2 \%(31 / 337)$. The patients with prestroke cognitive decline were older, more often had less than 6 years of education, and had history of previous stroke. Vascular risk factors did not differ significantly between these two groups. White matter changes $(p=0.004)$, cortical entorhinal, hippocampal, and medial temporal atrophy $(\mathbf{p}<0.001)$, cortical frontal atrophy $(p=0.008)$; and any central atrophy $(p<0.01)$, but not the frequencies or volumes of old, silent, or all infarcts on MRI differentiated those with and without prestroke cognitive decline. The correlates of prestroke cognitive decline in logistic regression analysis were medial temporal cortical atrophy (odds ratio (OR) 7.5, 95\% confidence interval $(95 \% \mathrm{CI}) 3.2-18.2)$, history of previous ischaemic stroke (OR 4.4, 95\% CI 1.8-10.6), and education (OR $0.9,95 \%$ CI $0.8-0.9)$.

Conclusions-History of previous stroke, but not volumes or frequencies was found to correlate with prestroke cognitive decline. Other associating factors were rather those usually associated with degenerative dementia: white matter changes and cerebral atrophy; and in multiple models medial temporal cortical atrophy and education. The possible over- lap between two or more underlying diseases must be remembered in diagnosis and treatment of patients with vascular cognitive impairment.

(F Neurol Neurosurg Psychiatry 1999;67:742-748)

Keywords: stroke; cognition; MRI

Cerebrovascular disease is associated with higher risk of dementia, and vascular factors are related to other causes of dementia. ${ }^{1-4}$ Stroke seems to be related to dementia more often than previously assumed ${ }^{5}$ and vascular factors are also related to Alzheimer's disease. ${ }^{6}$ These factors include white matter changes, ${ }^{2-8}$ cerebral amyloid angiopathy, ${ }^{9-11}$ and coexisting stroke. ${ }^{12}$ Stroke, risk factors for stroke, and Alzheimer's disease share common genetic factors, such as the $\in 4$ allele of the apolipoprotein E gene. ${ }^{13-16}$ In conclusion, it has been found that vascular factors are more often related to dementia and cognitive impairment in Alzheimer's disease, than has previously been assumed.

The prevalence of dementia is increased after an ischaemic stroke. ${ }^{4121718}$ According to modified DSM-III criteria, Tatemichi et al found that $26.3 \%$ of stroke patients were demented 3 months after ischaemic stroke, ${ }^{12}$ $34.6 \%$ were considered to have Alzheimer's disease with stroke, because functional and memory impairments preceded the index stroke. ${ }^{12}$ In the Helsinki Stroke Aging Memory Study cohort baseline findings, nearly $40 \%$ of patients found demented 3 months poststroke had cognitive decline before stroke. ${ }^{19}$ Kase et $a l^{20}$ assessed the cognitive performance (mini mental state examination (MMSE)) of 74 subjects from the Framingham Study cohort, who had had a stroke during a 13 year period. Comparing prestroke and poststroke cognitive performance, they found that the patients with stroke had a significantly lower mean MMSE score at prestroke baseline, compared with control subjects. A significant decline in mean MMSE score took place poststroke. The finding of lower prestroke MMSE remained unexplained in this study. ${ }^{20}$ Recently, Henon et al ${ }^{1}$ reported that the frequency of prestroke dementia in a series of stroke patients (mean age 75 ) was $16.3 \%(33 / 202)$ and in logistic regression analysis female sex, family dementia, leukoaraiosis, and cerebral atrophy were independently associated with prestroke dementia

These findings emphasise that the pathophysiology of poststroke dementia includes ischaemic changes in the brain, a combination 
of degenerative and vascular changes, and changes only related to Alzheimer's disease. Therefore some cognitive decline recognised after a stroke may be due to pre-existing decline.

The aim of the present study was to determine the frequency of prestroke cognitive decline by standardised questionnaires of patients' relatives and to determine the radiological and clinical correlates of it.

\section{Subjects and methods}

Procedures of the Helsinki Stroke Aging Memory Study cohort have been detailed in a report on methods and baseline findings. ${ }^{19}$ Briefly, 486 consecutive patients aged 55 to 85 were evaluated 3 months after ischaemic stroke. Of these, 451 completed a clinical, neurological, and mental status examination. ${ }^{19}$ The excluded patients comprised one who refused, one with a reduced level of consciousness, one with severe hearing impairment, and 32 with severe aphasia. Of the 451 patients, $337(74.7 \%)$ had brain MRI and a comprehensive neuropsychological examination. The 114 patients not included were 59 in whom MRI was not performed (contraindication in 27, refusal in 18 , claustrophobia in two, severe illness in 11 , obesity in one) and 55 who did not complete the cognitive test battery in detail (lack of sufficient cooperation in 23, refusal in 14 , non-fluency in the Finnish language in five, severe aphasia in five, severe neglect in three, hearing or visual impairment in three, illiteracy in two). The 114 patients excluded were older (mean age, $73.5 \vee 70.2$ years; $p<0.001$ ), less often had small vessel occlusion (1/114 v $27 / 337 ; p=0.0064)$, and more often stroke of undetermined aetiology (82/114 versus 202/ $337 ; \mathrm{p}=0.0219)$, but they did not differ in terms of main demographic and clinical features including sex, education, and number, side, and site of stroke).

The subjects underwent a structured medical and neurological history based on review of all available hospital charts, interview of the subject and a knowledgeable informant, and a structured clinical and neurological examination performed by a board certified neurologist (TP). In addition, all the cases were reviewed by a senior neurologist (TE). The neurological examination focused on factors and features related to dementia and stroke similar to the method of the Memory Research Unit, Department of Neurology, University of Helsinki, ${ }^{22}$ and the National Stroke Data Bank. ${ }^{23}$ History of main vascular risk factors was obtained as described earlier. ${ }^{19}$

Details of the clinical mental status examination, as well as assessment of emotional functions, are given in a previous report. ${ }^{19}$ The clinical cognitive assessment included the Folstein MMSE (maximum 30) ${ }^{24}$ Aphasia was assessed clinically using the acute aphasia screening protocol. ${ }^{25}$ Assessment of emotional functions included the Beck depression scale completed by the patient (maximum $=60) .{ }^{26}$ The neuropsychological criteria for dementia were those of the American Psychiatric Association DSM-III. ${ }^{27}$ Cognitive domains of the
DSM-III definition assessed by the neuropsychological test battery included memory functions (short and long term memory), executive functions, abstract thinking, judgment, aphasia, apraxia, agnosia, constructional and visuospatial abilities, and personality change. The detailed description of the assessment has been published. ${ }^{28}$

Social functioning was assessed by the patient's ability to work, by the patient's ability to perform the instrumental activities of daily living (IADL) and activities of daily living (ADL) tests based on an interview with the patient and with a knowledgeable informant, and by neurologist's examination. Assessments that reflected functions before and 3 months after the index stroke were used. The scales used included the index of ADL (rating from 1 to 7 ), ${ }^{29}$ the IADL scale (maximum $=8$ ), ${ }^{30}$ the functional activities questionnaire (maximum $=30),{ }^{31}$ the Blessed functional activity scale (maximum $=17),{ }^{32}{ }^{33}$ and the Barthel index. ${ }^{34}$ In addition, the neurologist completed the clinical dementia rating (maximum $=3$ ). ${ }^{35}$ Stroke related impairment was also assessed using the Rankin scale (maximum $=5)^{36}$ and the Scandinavian stroke scale (maximum $=58$ ).$^{37}$

Assessment of prestroke cognitive decline was based on interviews with the patient and a knowledgeable informant. The interviews included structured questions on abnormality in the cognitive domains as well as assessment of social functions before the index stroke. The beginning of the symptoms and their duration was asked for; the focus was especially in the period of 1 year before the index stroke. We used all available history from the patient and knowledgeable informant to get the most reliable answers to all the structured questions and based on the questionnaire, a board certified neurologist (TP) independently judged whether the patient had prestroke cognitive decline or not. All the cases were further reviewed by a senior neurologist (TE) and because we took only patients with clear cognitive impairment, there was no disagreement between the two neurologists. The patients also included those with borderline and definite dementia. However, we were unable to perform structured diagnosis of prestroke dementia; the focus was on prestroke cognitive decline. Our definiton accords with that of Tatemichi et al. ${ }^{38}$

Education was divided into two categories: low (0 to 6 years of formal education) and high ( $>6$ years of formal education).

MAGNETIC RESONANCE IMAGING

Brain MRI was performed 3 months after index stroke with a superconducting MRI system operating at $1.0 \mathrm{~T}$ (Siemens Magnetom, Erlangen, Germany). Transaxial T2, PD, and T1 weighted images were obtained with the SE technique. The repetition time (TR) for T2 and PD weighted images was $3000 \mathrm{~ms}$, echo time (TE) 15-90 ms, and number of excitations (NEX) 1. The corresponding parameters for $\mathrm{T} 1$ weighted images were $\mathrm{TR} / \mathrm{TE} / \mathrm{NEX}$ $400 / 15 / 2$. The slice thickness was $5 \mathrm{~mm}$, gap 0 , field of view $230 \mathrm{~mm}$, matrix size $256 \times 256$ 
pixels, and number of slices 26 on every pulse sequence. In addition a three dimensional gradient echo TR/TE/alpha/NEX 30/5/40/1 -sequence with $643 \mathrm{~mm}$ thick coronal sections was used.

BRAIN INFARCTS

All MR images were reviewed by the same neuroradiologist (RM) blinded to the clinical data. The number, location, and size of focal lesions were recorded. Lesions equivalent to the signal characteristics of CSF on T1 weighted images and measuring over $3 \mathrm{~mm}$ in diameter, as well as wedge shaped corticosubcortical lesions were regarded as brain infarcts. The size of the lesions was allocated to four groups: diameter 3-9 mm, 10-29 mm, 30-59 $\mathrm{mm}$, and $\geqslant 60 \mathrm{~mm}$, and the radii used for calculations were $3,10,20$, and $30 \mathrm{~mm}$ respectively. The volume of the lesion was estimated using the formula for calculating the volume of a sphere.

The history of previous and present (index stroke) including clinical symptoms and radiological lesions on MRI were reviewed together with the board certified neurologist (TP) and neuroradiologist $(\mathrm{RM})$. The infarcts not related to the present symptoms, but which were related to episodes documented in the patient's history, were defined as old infarcts. The infarcts not having any relation to the patient's present or previous symptoms were defined as silent infarcts.

WHITE MATTER CHANGES

For white matter hyperintensity (WMHI) rating nine slices $(\mathrm{L} 1, \mathrm{~L} 2, \mathrm{~L} 3, \mathrm{H} 1, \mathrm{H} 2, \mathrm{H} 3, \mathrm{~S} 1$, S2, S3) were chosen to represent the different parts of white matter. Slice L1 was the lowest slice where the frontal horns of lateral ventricles could be seen and S1 the first slice above the roof of the lateral ventricles. ${ }^{39}$

White matter hyperintensities were rated in four white matter areas: periventricular, deep, watershed, and subcortical white matter. Periventricular hyperintensities (PVHIs) were in contact with the ventricular wall; deep WMHIs (DWMHIs) were separated from the ventricular system by a strip of normal looking white matter and were located outside the watershed. The subcortical region was considered to represent the area $<5 \mathrm{~mm}$ beneath the cortex. White matter hyperintensities which affected some part of this area and were located outside watershed were rated as subcortical HIs. Watershed HIs and PVHIs were analysed on six slices (L1, L2, L3, H1, H2, H3); DWMHs on three slices (S1, S2, S3); and subcortical HIs on nine slices (L1, L2, L3, H1, H2, H3, S1, S2, S3). ${ }^{39}$

Periventricular hyperintensities (PVHIs) around the frontal and posterior horns were classified based on size and shape into small cap, large cap, and extending cap (small cap $\leqslant$ $5 \mathrm{~mm}$ in diameter, rounded with regular margins; large cap 6 to $10 \mathrm{~mm}$ in diameter, mostly with regular margins; extending cap $>10 \mathrm{~mm}$ in diameter, irregular margins). Periventricular hyperintensities along the bodies of lateral ventricles were classified based on thickness and shape into thin lining, smooth halo and irregular halo (thin lining $\leqslant 5 \mathrm{~mm}$, regular margins; smooth halo 6 to $10 \mathrm{~mm}$, smooth and mostly regular margins; irregular halo $>10 \mathrm{~mm}$, irregular margins and extending into the deep white matter).

White matter hyperintensities in the subcortical, deep, and watershed areas were classified based on size (greatest diameter) and shape into small focal, large focal, focal confluent, diffusely confluent, and extensive white matter change (small focal, punctate hyperintensities $\leqslant 5 \mathrm{~mm}$, mostly rounded; large focal 6 to 10 $\mathrm{mm}$, mostly rounded; focal confluent 11 to 25 $\mathrm{mm}$, often various shapes and may have irregular borders; diffusely confluent $>25 \mathrm{~mm}$, mostly irregular borders; and extensive white matter change, diffuse hyperintensity without distinct focal lesions affecting most of the white matter area). The number of each type of hyperintensity was counted, and extensive white matter change was rated as absent or present. ${ }^{39} 40$

The extent of PVHIs and WMHIs on PD weighted images was then graded in the following way using a four point scale proposed by Fazekas et $a l^{41}$ : PVHI grade $0=$ absence of PVHIs, $1=$ small or large caps or thin lining, $2=$ smooth halo, $3=$ extending caps or irregular halo; WMHI grade $0=$ absence of WMHIs, $1=$ small or large focal lesions only, $2=$ at least one focal confluent lesion, no diffusely confluent lesions nor extensive white matter change, $3=$ at least one diffusely confluent lesion or extensive white matter change.

\section{BRAIN ATROPHY}

Brain atrophy was rated from 0 to 3 corresponding to atrophy ratings "no, mild, moderate, and severe". Ratings were made by comparison to standard images in the following way: grade $0=$ none $=$ equal to standard image 0 or less than in standard image 1; grade $1=$ mild=equal to standard 1 or less than standard 2; grade $2=$ moderate $=$ equal to standard 2 or less than standard 3; and grade $3=$ severe $=$ equal to standard 3 or more than in standard images. Cortical and central brain atrophy were rated separately; cortical atrophy in the frontal, parietal, and occipital lobes, in the temporal neocortex, entorhinal cortex (parahippocampal gyrus), and hippocampal formation as well as in the cerebellum and vermis, and central atrophy in the temporal, frontal, and occipital horns of the lateral ventricles as well as the bodies, in the third and fourth ventricles. If possible, both sides were rated separately. The atrophy ratings were based on T1 weighted images and the temporal structures were evaluated on three coronal slices, (the slice showing the interpeduncular cistern and \pm 1 slice). In the analysis of cortical atrophy, attention was paid to the width of sulci and the thickness of gyri. In addition to general cortical atrophy, focal cortical atrophy in the frontal, parietal, occipital or temporal lobes, as well as the cerebellum was registered. 
Table 1 Characteristics of patients without and with cognitive decline prestroke in the Helsinki Stroke Aging Memory Study (SAM) Cohort $(n=337)$

\begin{tabular}{lllll}
\hline Characteristic & $\begin{array}{l}\text { No cognitive decline } \\
\text { prestroke }(n=306)\end{array}$ & $\begin{array}{l}\text { Cognitive decline } \\
\text { prestroke }(n=31)\end{array}$ & All $(n=337)$ & $p$ Value \\
\hline Mean age (SD) & $69.9(7.4)$ & $72.9(9.9)$ & $70.2(7.7)$ & 0.0363 \\
Female sex (\%) & $149(48.7)$ & $19(61.3)$ & $168(49.9)$ & 0.1813 \\
Low education (\%) & $78(26.2)$ & $14(45.2)$ & $92(28.0)$ & 0.0250 \\
Risk factor for stroke: & & & $165(49.0)$ & 0.7566 \\
$\quad$ Arterial hypertension & $149(48.7)$ & $16(51.6)$ & $79(23.4)$ & 0.5729 \\
$\quad$ Diabetes & $73(23.9)$ & $6(19.4)$ & $69(20.5)$ & 0.0899 \\
$\quad$ Cardiac failure & $48(19.3)$ & $10(32.3)$ & $56(16.6)$ & 0.1492 \\
$\quad$ Atrial fibrillation & $59(19.3)$ & $8(25.8)$ & $62(18.4)$ & 0.1885 \\
$\quad$ History of myocardial infarction & $56(18.3)$ & $3(9.7)$ & $59(17.5)$ & 0.2286 \\
$\quad$ High total cholesterol & $157(51.3)$ & $13(4)$ & $170(50.5)$ & 0.3200 \\
$\quad$ Smoking; history or current & $11(26.6)$ & $9(29.0)$ & $90(26.8)$ & 0.7669 \\
$\quad$ Alcohol use (at least once a week) & $17(5.6)$ & $0(0.0)$ & $17(5.1)$ & 0.1766 \\
$\quad$ None of the above & $42(13.7)$ & $2(6.5)$ & $44(13.1)$ & 0.2520 \\
History of previous CVD: & $55(18.0)$ & $15(48.4)$ & $70(20.8)$ & 0.0000 \\
Transient ischaemic attack & $4(1.3)$ & $1(3.2)$ & $5(1.5)$ & 0.3998 \\
Ischaemic stroke & $3(1.0)$ & $0(0.0)$ & $3(0.9)$ & 0.5797 \\
Subarachnoidal haemorrhage & & & \\
Intracerebral haemorrhage & & &
\end{tabular}

RELIABILITY OF RATING

Reliability of the visual rating was tested by reviewing $60 \mathrm{MR}$ scans independently by the same rater (RM), by a board certified neuroradiologist (OS), and a general radiologist (HJA). The weighted $\kappa$ values for intraobserver agreement were 0.90 for periventricular caps, 0.93 for linings and halos, and 0.95 for DWMHIs. The corresponding $\kappa$ values for interobserver agreement were $0.82-0.84$ for caps, $0.72-0.82$ for linings and halos, and $0.77-0.84$ for DWMHIs. ${ }^{39}$ The intraobserver reliability for atrophy assessment was 0.80 for temporal neocortical atrophy, 0.75 for atrophy in entorhinal cortex, and 0.82 for hippocampal atrophy. The corresponding interobserver reliability was 0.71-0.74 for neocortical, $0.63-0.69$ for entorhinal, and 0.61-0.62 for hippocampal atrophy as expressed by weighted $\kappa$ scores.

The study was approved by the ethics committee of the Department of Neurology, University of Helsinki. It was first fully explained to the patients, and if they agreed to participate, a written consent form was signed.

We compared the patients with and without prestroke cognitive decline. The $\chi^{2}$ test was applied for categorical data and the pooled $t$ test for continuous data. All the variables that significantly differentiated the two groups were put into a logistic regression model to work out the correlates of prestroke cognitive decline.
The statistics were analysed using the BMDP and SAS programs. ${ }^{42} 43$

\section{Results}

Of the 337 patients with ischaemic stroke, prestroke cognitive decline was present in 31 $(9.2 \%)$. The patients with prestroke cognitive decline were older and more often had less than 6 years of education (table 1 ).

Vascular risk factors did not differ significantly between those with and without prestroke cognitive decline (table 1). History of previous ischaemic stroke $(48.4 \%$ v $18.0 \%$ : $\mathrm{p}<0.0001)$, but not that of transient ischaemic attacks $(6.5 \%$ v $13.7 \% ; \mathrm{p}=0.2520)$ were more frequent in the patients with prestroke cognitive decline than in those without it (table 1).

The degree of moderate and severe white matter changes and cerebral atrophy is presented in table 2. Mean Fazekas white matter score of those with prestroke cognitive decline was significantly higher compared with those without prestroke cognitive decline $(4.0(1.7) v$ 3.1 (1.5); $\mathrm{p}=0.0037)$. Moderate and severe cortical frontal $(62.1 \% v 36.8 \% ; \mathrm{p}=0.0078)$, entorhinal $(69.0 \%$ v $21.0 \% ; \mathrm{p}<0.0001)$, hippocampal $(55.2 \%$ v $15.5 \% ; \mathrm{p}<0.0001)$, and medial temporal atrophy $(69.0 \%$ v $22.0 \%$; $\mathrm{p}<0.0001$ ) were significantly more common among those with than those patients without prestroke cognitive decline. Moderate and

Table 2 Percentages of moderate and severe degree of white matter changes and cerebral atrophy in patients with and without prestroke cognitive decline in the Helsinki Stroke Aging Memory (SAM) Study Cohort ( $n=337)$

\begin{tabular}{|c|c|c|c|c|}
\hline & $\begin{array}{l}\text { No cognitive decline } \\
\text { prestroke }(n=306)\end{array}$ & $\begin{array}{l}\text { Cognitive decline } \\
\text { prestroke }(n=31)\end{array}$ & All $(n=337)$ & $p$ Value \\
\hline \multicolumn{5}{|l|}{ White matter changes: } \\
\hline Periventricular & 52.5 & 72.4 & 54.3 & 0.0404 \\
\hline Centrum semiovale & 20.8 & 44.8 & 22.9 & 0.0032 \\
\hline Watershed areas & 32.2 & 48.3 & 33.6 & 0.0805 \\
\hline Subcortical & 8.1 & 6.9 & 8.0 & 0.8147 \\
\hline Mean Fazekas white matter score (SD) & $3.1(1.5)$ & $4.0(1.7)$ & $3.2(1.5)$ & 0.0037 \\
\hline \multicolumn{5}{|l|}{ Cortical atrophy: } \\
\hline Frontal & 36.8 & 62.1 & 39.1 & 0.0078 \\
\hline Parietal & 41.2 & 58.6 & 42.3 & 0.0706 \\
\hline Occipital & 41.2 & 48.3 & 41.9 & 0.4620 \\
\hline Entorhinal & 21.0 & 69.0 & 25.2 & 0.0000 \\
\hline Hippocampal & 15.5 & 55.2 & 19.1 & 0.0000 \\
\hline Medial temporal & 22.0 & 69.0 & 26.2 & 0.0000 \\
\hline \multicolumn{5}{|l|}{ Central atrophy: } \\
\hline Temporal & 28.0 & 65.5 & 31.4 & 0.0000 \\
\hline Frontal & 35.1 & 65.5 & 37.1 & 0.0013 \\
\hline Lateral & 38.9 & 65.5 & 41.2 & 0.0054 \\
\hline Occipital & 41.9 & 75.9 & 44.9 & 0.0004 \\
\hline
\end{tabular}


Table 3 Frequency (SD) and mean volumes ( $\mathrm{cm}^{3}$, SD) of infarcts on MRI in patients without and with cognitive decline prestroke in the Helsinki Stroke Aging Memory Study (SAM) Cohort ( $n=337)$

\begin{tabular}{|c|c|c|c|c|c|c|}
\hline & \multicolumn{2}{|l|}{ Old infarcts } & \multicolumn{2}{|l|}{ Silent infarcts } & \multicolumn{2}{|l|}{ All } \\
\hline & $\begin{array}{l}\text { No cognitive decline } \\
\text { prestroke }(n=306)\end{array}$ & $\begin{array}{l}\text { Cognitive decline } \\
\text { prestroke }(n=31)\end{array}$ & $\begin{array}{l}\text { No cognitive decline } \\
\text { prestroke }(n=306)\end{array}$ & $\begin{array}{l}\text { Cognitive decline } \\
\text { prestroke }(n=31)\end{array}$ & $\begin{array}{l}\text { No cognitive decline } \\
\text { prestroke }(n=306)\end{array}$ & $\begin{array}{l}\text { Cognitive decline } \\
\text { prestroke }(n=31)\end{array}$ \\
\hline \multicolumn{7}{|c|}{ Frequency of infarcts: } \\
\hline Right side & $0.2(0.9)$ & $\begin{array}{l}0.5(1.0) \\
\mathrm{p}=0.0743\end{array}$ & $0.8(1.2)$ & $\begin{array}{l}0.8(1.0) \\
\mathrm{p}=0.8798\end{array}$ & $1.5(0.1)$ & $\begin{array}{l}1.5(0.3) \\
\mathrm{p}=0.6690\end{array}$ \\
\hline Left side & $0.3(1.0)$ & $\begin{array}{l}0.2(0.6) \\
\mathrm{p}=0.6403\end{array}$ & $0.7(1.0)$ & $\begin{array}{l}0.7(0.9) \\
\mathrm{p}=0.8008\end{array}$ & $1.4(1.4)$ & $\begin{array}{l}1.3(1.9) \\
\mathrm{p}=0.2248\end{array}$ \\
\hline All & $0.6(1.4)$ & $\begin{array}{l}0.8(1.1) \\
\mathrm{p}=0.4100\end{array}$ & $1.5(1.8)$ & $\begin{array}{l}1.5(1.5) \\
\mathrm{p}=0.9608\end{array}$ & $3.0(2.4)$ & $\begin{array}{l}3.2(2.0) \\
\mathrm{p}=0.5795\end{array}$ \\
\hline \multicolumn{7}{|c|}{ Volume of infarcts: } \\
\hline Right side & $20.4(33.5)$ & $\begin{array}{l}17.8(37.7) \\
\mathrm{p}=0.8405\end{array}$ & $2.8(6.7)$ & $\begin{array}{l}4.9(8.4) \\
p=0.2731\end{array}$ & $22.8(38.5)$ & $\begin{array}{l}32.6(51.3) \\
\mathrm{p}=0.2426\end{array}$ \\
\hline Left side & $10.6(21.8)$ & $\begin{array}{l}2.6(2.3) \\
\mathrm{p}=0.4250\end{array}$ & $3.5(9.4)$ & $\begin{array}{l}0.8(1.5) \\
\mathrm{p}=0.2711\end{array}$ & $11.4(21.3)$ & $\begin{array}{l}10.6(26.4) \\
p=0.8813\end{array}$ \\
\hline All & $17.2(29.8)$ & $\begin{array}{l}14.4(32.7) \\
\mathrm{p}=0.7714\end{array}$ & $4.5(10.2)$ & $\begin{array}{l}4.0(7.3) \\
p=0.8235\end{array}$ & $26.5(39.4)$ & $\begin{array}{l}36.7(50.7) \\
\mathrm{p}=0.2049\end{array}$ \\
\hline
\end{tabular}

severe central temporal $(65.5 \%$ v $28.0 \%$; $\mathrm{p}<0.0001)$, frontal $(65.5 \%$ v $35.1 \%$; $\mathrm{p}=0.0013)$, lateral $(65.5 \% \quad v \quad 38.9 \%$; $\mathrm{p}=0.0054)$, and occipital $(75.9 \%$ v $41.9 \%$; $\mathrm{p}=0.0004$ ) atrophy were significantly more common among the patients with prestroke cognitive decline than the patients without it (table 2).

Mean frequencies and volumes $\left(\mathrm{cm}^{3}\right)$ of old, silent, and all infarcts are presented in table 3. The mean frequencies and volumes of old, silent and all infarcts, right and left sided and all analysed separately, did not significantly differentiate the patients with cognitive decline from those without it.

The variables that significantly differentiated the patients with and without prestroke cognitive decline were put into a multiple logistic model (table 4). Prestroke cognitive decline was related to medial temporal cortical atrophy (odds ratio (OR) 7.5, 95\% confidence interval $(95 \% \mathrm{CI}) 3.2-18.2)$, to history of previous ischaemic stroke (OR 4.4, 95\% CI 1.8-10.6) and to education (OR $0.9,95 \%$ CI $0.8-0.9$ ).

Three month outcome was worse in patients with prestroke cognitive decline compared with those without measured by all ADL scales and

Table 4 Correlates of prestroke cognitive decline in the Helsinki Stroke Aging Memory Study (SAM) Cohort using multiple logistic models

\begin{tabular}{lllll}
\hline Variable & $B$ & $S E$ & OR & $95 \% C I$ \\
\hline Medial temporal cortical atrophy & 2.0177 & 0.4378 & 7.5 & $3.2-18.2$ \\
History of previous ischaemic stroke & 1.4748 & 0.4429 & 4.4 & $1.8-10.6$ \\
Education & -01436 & 0.0644 & 0.9 & $0.8-0.9$ \\
Constant & -3.7496 & & & \\
\hline
\end{tabular}

$\mathrm{B}=$ Coefficient; $\mathrm{SE}=$ standard error.

Table 5 Three month outcome according to prestroke cognitive decline

\begin{tabular}{lllll}
\hline & $\begin{array}{l}\text { No cognitive } \\
\text { decline prestroke } \\
(n=306)\end{array}$ & $\begin{array}{l}\text { Cognitive } \\
\text { decline } \\
\text { prestroke } \\
(n=31)\end{array}$ & All $(n=337)$ & p Value \\
\hline Variable & $55.7(5.5)$ & $53.4(6.7)$ & $55.4(5.7)$ & 0.0335 \\
SSS (SD) & $1.3(0.8)$ & $2.2(1.6)$ & $1.4(1.0)$ & 0.0000 \\
Index of ADL (1-7) & $6.3(2.1)$ & $3.7(2.6)$ & $6.1(2.2)$ & $<0.0001$ \\
IADL (0-8) & $5.1(7.0)$ & $16.8(10.6)$ & $6.2(8.1)$ & 0.0000 \\
FAQ (0-30) & $1.1(1.9)$ & $4.5(3.1)$ & $1.4(2.3)$ & $<0.0001$ \\
BFAS (0-17) & $74.6(11.3)$ & $65.7(19.5)$ & $73.8(12.5)$ & 0.0002 \\
Barthel index (0-100) & $1.9(1.0)$ & $2.9(1.0)$ & $2.0(1.0)$ & $<0.0001$ \\
Rankin (SD) & $0.4(0.4)$ & $1.0(0.6)$ & $0.5(0.4)$ & $<0.0001$ \\
CDR (SD) & $87(28.4)$ & $20(64.5)$ & $107(31.8)$ & $<0.0001$ \\
DSM-III-Dementia (\%) & $26.7(2.8)$ & $22.4(4.5)$ & $26.3(3.2)$ & $<0.0001$ \\
MMSE (SD) & $9.0(7.1)$ & $12.4(8.5)$ & $9.3(7.3)$ & 0.0139 \\
Beck (SD) & $49.1(4.3)$ & $46.6(4.4)$ & $48.9(4.4)$ & 0.0020 \\
AASP & & & & \\
\hline
\end{tabular}

cognitive scales (table 5). The amount of patients found demented 3 months after stroke was significantly higher in those patients with prestroke cognitive decline compared with those without $(20(64.5 \%)$ v 87 (28.4\%); $\mathrm{p}<0.0001)$.

\section{Discussion}

We report here the largest well defined stroke cohort thus far to examine the correlates related to prestroke cognitive decline. Based on interviews with the patient and a knowledgeable informant, prestroke cognitive decline including patients with dementia, was found in $9.2 \%$ of the patients (mean age 70.2 ) with ischaemic stroke. In an earlier study ${ }^{21}$ prestroke dementia was found in $16.3 \%$ of the patients with stroke (mean age 75 ) by structured questionnaires of the relatives. In meta-analysis of nine European studies ${ }^{43}$ age specific prevalence (\%) of pre-existing dementia in the groups aged 40 to 69,70 to 79 and $\geqslant 80$ years was $4.4 \%, 9.7 \%$, and $33.3 \%$, respectively in stroke patients. The corresponding prevalence in patients with dementia (whatever the aetiology) in community based studies were $0.5 \%$, $5.0 \%$, and $19.1 \%{ }^{44}$ Thus in the present study we think that we have avoided overestimation of this prevalence. We were able to investigate in detail (including brain MRI) $75 \%$ of the clinically testable patients. The nonparticipants were older and more often had stroke of undetermined aetiology. This factor might have caused a slight underestimation of the frequency of prestroke cognitive impairment. Altogether $11.8 \%$ of all the clinically testable patients had prestroke cognitive imapairment and in the present approach a little less, $9.2 \%$ of the patients had prestroke cognitive imapairment and also MRI. However, this selection bias is comparable with that in other similar cohorts. ${ }^{12} 38$

Previous stroke, but not transient ischaemic attack or any known risk factor for stroke, old, silent, or all infarcts on MRI, was associated with prestroke cognitive decline. Cerebral atrophy and white matter changes were strongly associated with prestroke cognitive decline. In the logistic model the independent correlates of prestroke cognitive decline were medial temporal cortical atrophy, history of previous stroke, and education. In an earlier study ${ }^{21}$ 
factors independently associated with preexisting dementia were those usually associated with degenerative dementia rather than those associated with vascular dementia: cerebral atrophy, leukoaraiosis, family history of dementia, and female sex. In that study previous stroke, transient ischaemic attack, silent infarcts, or the presence of old infarcts on CT were not associated with pre-existing dementia.

Assessment of prestroke cognitive decline was based on interviews with the patient and a knowledgeable informant. The interview included structured questions on abnormality in the cognitive domains as well as assessment of social functions before the index stroke. This kind of evaluation has certain limits, because it is based on a retrospective questionnaire, but has been used in earlier studies in structured interviews used for epidemiological studies for patients still alive ${ }^{45}$ or even after a patient's death, ${ }^{46}$ and also in stroke patients. ${ }^{21}{ }^{38}$ The information from the relatives in the diagnosis of dementia has proved useful also when recorded in the unstructured manner of the clinical history. ${ }^{47}{ }^{48}$ Being aware of the limitations of our evaluation, we are satisfied with the definition of prestroke cognitive decline instead of dementia.

Current epidemiological, clinical, and neuropathological evidence points to a substantial overlap between Alzheimer's disease and vascular dementia and suggests that vascular pathology, the traditional cornerstone of the differential diagnosis between the two entities, may not represent as clear a line of demarcaration as originally thought. ${ }^{49}$ These two types of dementia (Alzheimer's disease and vascular dementia) have some possible common features that are responsible for this overlap. In our study white matter changes were associated with prestroke cognitive decline. White matter changes have been found to overlap considerably between the subcortical subtype of vascular dementia and Alzheimer's disease with late onset (type II). ${ }^{50}$ In the second, white matter changes and Alzheimer encephalopathy may interact to produce dementia. The history of previous stroke was independently associated with prestroke cognitive decline, also reflecting the vascular mechanisms behind it. However, the number or volumes of old, silent, or all infarcts in MRI were not associated with prestroke cognitive decline in the present study.

Medial temporal cortical atrophy was the factor having independent and strongest association with prestroke cognitive decline in our study. In earlier studies, cortical atrophy has been found to be a strong determinant of prestroke dementia ${ }^{21}$ and also poststroke dementia, ${ }^{17}$ which may partly be explained by normal aging effects. ${ }^{51}$ In a study conducted on 170 consecutive stroke patients, Henon et $a \tilde{l}^{2}$ found that patients with medial temporal lobe atrophy are more likely to have pre-existing dementia. This suggests that Alzheimer's disease might contribute to the dementia syndrome.

Pre-existing cognitive decline also influenced the 3 month outcome in terms of all ADL scales and scales measuring handicap after stroke (table 5). Also cognitive decline poststroke was significantly worse in those patients with prestroke cognitive decline compared with those without, measured by MMSE, CDR and in amount of patients found demented by DSM-III criteria. The correlates of dependent living 3 months after ischaemic stroke in this cohort have been published. ${ }^{53}$ The Rankin scale explained $51.5 \%$, functional activities questionnaire $5.9 \%$, and presence of dementia or any poststroke cognitive decline $3.4 \%$ of the total variance between dependent and independent patients after stroke, so prestroke cognitive decline did not have an independent correlation with dependence poststroke. This is in agreement with the findings of Henon et $a l^{21}$ who did not find pre-existing dementia to influence the very short term prognosis poststroke.

Cognitive deficits can be detected several years before the clinical diagnosis of dementia. $^{54}$ The neuropsychological profile may indicate the underlying neuropathology, but there may be overlap between two or more diseases, which complicates the diagnostic process. ${ }^{54}$ Recognition of degenerative pathology in a patient with poststroke dementia is important because of the emergence of effective treatments for Alzheimer's disease. ${ }^{55}$ It seems that the prevention of stroke offers the most immediate and substantial solution to reduce the morbidity and mortality associated with vascular dementia. ${ }^{56}$ Once vascular dementia occurs, control of risk factors for stroke and the use of conventional stroke preventives may be useful. Pentoxifylline, a haemorheological agent, ${ }^{57}$ and propentofylline, an adenasine uptake/phosphodiesterase inhibitor with neuroprotective properties, ${ }^{58}$ have shown promise in protecting the brain from ischaemia. Donepezil, approved for use in Alzheimer's disease, could be effective in vascular dementia, especially if there is mixed vascular dementia and Alzheimer's disease. ${ }^{59}$

The prevalence of both stroke and Alzheimer's disease increase with age, and vascular disease may be an important predictor of the presence and severity of cognitive impairment in older people. Kuller et al demonstrated that vascular changes on MRI, measures of brain atrophy, ApoE-4, and age, education, and race are associated with low cognitive scores among older people. ${ }^{60}$ An important precursor to preventive and treatment strategies for vascular dementia is diagnostic consensus. This needs to be established in the areas of diagnostic criteria for dementia in general and for vascular dementia and cognitive decline in particular. Some cognitive decline recognised after a stroke seems to be due to pre-exisitng decline. The possible overlap between two or more underlying diseases must be remembered in diagnosis, treatment, and development of new treatments to patients with vascular cognitive impairment.

This study was supported in part by grants from the Medical Council of the Academy of Finland (RM, HJA, TE); the Clinical Research Institute, Helsinki University Central Hospital (TP); the Finnish Alzheimer Foundation for Research, Helsinki (TP); the Finnish Alzheimer Foundation for Research, Helsinki
(TP, TE); the Paavo Nurmi Foundation, Helsinki, Finland 
(HJA); the Research Grant from the Helsinki University Central Hospital (HJA); Finnish Cultural Foundation (ML); and the Oscar Oflunds Stiftelse (ML). We thank research officer, Statistics Finland, Helsinki, for statistical support and review.

1 Tatemichi TK. How acute brain failure becomes chronic: a view of the mechanisms and syndromes of dementia related to stroke. Neurology 1990;40:1652-9.

2 Erkinjuntti T, Hachinski VC. Rethinking vascular dementia. Cerebrovasc Dis 1993;3:3-23.

3 Skoog I, Nilsson L, Palmertz B, et al. A population-based study on dementia in 85-year-olds. $N$ Engl $\mathcal{f} \mathrm{Med}$ 1993;328:153-8.

4 Kokmen E, Whisnat JP, O'Fallon WM, et al. Dementia after ischemic stroke: a population-based study in Rochester, Minnesota (1960-84). Neurology 1996;46:154-9.

5 Hachinski VC. Preventable senility: a call for action against the vascular dementias. Lancet 1992;340:645-8.

6 Skoog I, Lernfelt B, Landahl S, et al. The influence of white matter lesions on neuropsychological functioning in demented and non-demented 85-year-olds. Acta Neurol Scand 1996;93:142-8.

7 Breleter MB, van Amerongen NM, van Swieten JC, et al. Cognitive correlates of ventricular enlargement and cerebral white matter lesions on magnetic resonance imaging. ebral white matter lesions
Stroke 1994;25:1109-15.

8 Skoog I, Palmertz B, Andreasson L. The prevalence of white-matter lesions on computed tomography of the brain in demented and nondemented 85-year-olds. $\mathcal{F}$ Geriatr Psychiatry Neurol 1994;7:169-75.

9 Vinters HV. Cerebral amyloid angiopathy: a critical review. Stroke 1987;18:311-24.

10 Cohen DL, Hedera P, Premkumar DR, et al. Amyloid- $\beta$ protein angiopathies masquerading as Alzheimer's disease? Ann N Y Acad Sci 1997;826:390-5.

11 Kalaria $\mathrm{RN}$. Cerebrovascular degeneration is related to amyloid- $\beta$ protein deposition in Alzheimer's disease. Ann $N$ $Y$ acad Sci 1997;826:263-71.

12 Tatemichi TK, Desmond DW, Mayeux R, et al. Dementia after stroke: baseline frequency, risks, and clinical features in a hospitalized cohort. Neurology 1992;42:413.

13 Frisoni G, Geroldi C, Blanquetti A, et al. Apolipoprotein e4 allele frequency in vascular dementia and Alzheimer's disallele frequency in vascular dementia
ease [letter]. Stroke 1994;25:1703.

14 Gerdes LU. Apolipoprotein E genotypes and cardiovascular disease: a quantative overview of 42 studies. Genet Epidemiol 1994;11:294.

15 Saunders AM, Roses AD. Apolipoprotein E allele frequency, ischemic cerebrovascular disease, and Alzheimer's disease [letter]. Stroke 1993;24:1416.

16 Terry JG, Howard G, Mercuri M, et al. Apolipoprotein E polymorphism is associated with segment-specific extracra27:1755-9.

17 Tatemichi TK, Foulkes MA, Mohr JP, et al. Dementia in stroke survivors in the Stroke Data Bank cohort: prevalence, incidence, risk factors, and computed tomographic findings. Stroke 1990;21:858-66.

18 Loeb C, Gandolfo C, Croce R, et al. Dementia associated with lacunar infarction. Stroke 1992;23:1225-9.

19 Pohjasvaara T, Erkinjuntti T, Vataja R, et al. Dementia three months after stroke: baseline frequency and effect of different definitions of dementia in the Helsinki Stroke Aging Memory Study (SAM) cohort. Stroke 1998;24:35-41.

20 Kase CS, Wolf PA, Kelly-Hayes M, et al. Intellectual decline after stroke. Stroke 1998;29:805-12.

21 Henon H, Pasquir F, Durieu I, et al. Pre-exisitng dementia in stroke patients. Baseline frequency, associated factors, and outcome. Stroke 1997;28:2429-36.

22 Erkinjuntti T, Sulkava R, Kovanen J, et al. Suspected dementia: evalution of 323 consecutive referrals. Acta Neurol Scand 1987;76:359-64.

23 Foulkes MA, Wolf PA, Price TR, et al. The stroke data bank: design, methods, and baseline characteristics. Stroke 1988 19:547-54.

24 Folstein MF, Folstein SE, McHugh PR. Mini-mental state: a practical method for grading the cognitive state of patients for the clinician. F Psychiatr Res 1975;12:189-98.

25 Crary MA, Haak NJ, Malinsky AE. Preliminary psychometric evaluation of an acute aphasia screening protocol. ric evaluation of an acute

26 Beck AT, Beck RW. Screening depressed patients in family practice: Postgrad Med 1972;11:561-79.

27 American Psychiatric Association Committee on Nomenclature and Statistics. Diagnostic and statistical manual of mental disorders (DSM-III). 3rd ed. Washington, DC: APA, 1980.

28 Pohjasvaara T, Erkinjuntti T, Ylikoski R, et al. Clinical determinants of poststroke dementia. Stroke 1998;29:7581.

29 Katz S, Downs TD, Cash HR, et al. Progress in development of the index of ADL. Gerontologist 1970;8:2030.

30 Lawton MP, Brody EM. Assessment of older people: self-maintaining and instrumental activities of daily living. Gerontologists 1969;9:179-86.
31 Pfeffer RI, Kurosaki TT, Harrah CH, et al. Measurement of functional activities in older adults in the community. $\mathcal{F}$ functional activities in old $1982 ; 37: 323-9$.

32 Blessed G, Tomlinson BE, Roth $M$. The association between quantative measures of dementia and of senile change in the cerebral grey matter of elderly subjects. $\mathrm{Br} \mathcal{F}$ Psychiatry 1968;114:797-811.

33 Erkinjuntti T, Hokkanen L, Sulkava R, et al. The blessed dementia scale as a screening test for dementia. International fournal of Geriatric Psychiatry 1988;3:267-73.

34 Mahoney FI, Barthel DW. Functional evaluation: the Barthel index: a simple index of independence useful in scoring improvement in the rehabilitation of the chronically ill. Rehabilitation 1965:61-5.

35 Hughes CP, Berg L, Danziger WL, et al. A new clinical scale for the stating of dementia. Br F Psychiatry 1982;140:56672 .

36 Rankin J. Cerebral vascular accidents in patients over age of 60. Scott Med f 1957;2:200-15

37 Scandinavian Stroke Study Group. Multicenter trial of hemodilution in ischemic stroke: background and study protocol. Stroke 1985;16:885-90.

38 Tatemichi TK, Desmond DW, Stern Y, et al. Cognitive impairment after stroke: frequency, patterns, and relationship to functional abilities. F Neurol Neurosurg Psychiatry 1994;57:202-7.

39 Mäntylä R, Aronen HJ, Salonen O, et al. The prevalence and distribution of white matter changes on different MRI pilse sequences in a post-stroke cohort. Neuroradiology 1999; in

40 Mäntylä R, Erkinjuntti $\mathrm{T}$, Salonen $\mathrm{O}$, et al. Variable agreement between visual rating scales for white matter hyperintensities on MRI. Comparison of 13 rating scales in a poststroke cohort. Stroke 1997;28:1614-23.

41 Fazekas F, Chawluk JB, Alavi A, et al. MR signal abnormalities at $1.5 \mathrm{~T}$ in Alzheimer's dementia and normal aging. Am F Roentgenol 1987;149:351-6.

42 BMDP. New system for windows. Los Angeles, CA: BMDP: 1994.

43 SAS. SAS procedures guide, version 6, 3rd ed. Gary, NC: SAS Institute, 1990

44 Hofmann A, Rocca WA, Brayne C, et al, for the European Prevalence Research Group. The prevalence of dementia in Europe: a collaborative study of 1980-1990 findings. Int $\mathcal{F}$ Epidemiol 1991;20:736-48.

45 Copeland JRMC, Kelleher MJ, Kellet JM. A semistructured clinical interview for the assessment of diagnosis and mental state in the elderly. The Geriatric Mental State Schedule. I. Development and reliability. Psychol Med 1976;6:439-49.

46 Davis PB, White $\mathrm{H}$, Price JL, et al. Retrospective post-mortem dementia assessment: validation of a new clinical interview to assist neuropathologic study. Arch Neurol 1991;48:613-17.

47 Morris JC, Fulling K. Early Alzheimer's disease. Diagnostic considerations. Arch Neurol 1998;45:11-18.

48 Huppert FA, Tym E. Clinical and neuropsychological assessment of dementia. Br Med Bull 1986;42:11-18.

49 Gold G, Giannakopoulos P, Bouras C. Re-evaluating the role of vascular changes in the differential diagnosis of Alzheimer's disease and vascular dementia. Eur Neurol 1998;40:121-9.

50 Wallin A. The overlap between Alzheimer's disease and vascular dementia: the role of white matter changes. Dementia Geriatr Cogn Disord 1998;9 suppl 1:30-5.

51 Yerby MS, Sundsten JW, Larso EB, et al. A new method of measuring brain atrophy: the effect of aging and its application for diagnosing dementia. Neurology 1985;35: $1316-20$.

52 Henon H, Pasquier F, Durieu I, et al. Medial temporal lobe atrophy in stroke patients: relation to pre-existing dementia. F Neurol Neurosurg Psychiatry 1998;65:641-7.

53 Pohjasvaara T, Erkinjuntti T, Vataja R, et al. Correlates of dependent living 3 months after ischemic stroke. Cerebrovasc Dis 1998;8:259-66.

54 Pasquier F. Early diagnosis of dementia: neuropsychology. Neurol 1999;246;6-15.

55 Patel SV. Pharmacotherapy of cognitive impairment in Alzheimer's disease: a review. 7 Geriatr Psychiatry Neurol 1995;8:81-95.

56 Gorelick PB, Roman G, Mangone CA. Vascular dementia. In: Gorelick PB, Alter MA, eds. Handbook of neuroepidemiology. New York: Marcel Dekker, 1994:197-214.

57 European Pentoxifylline Multi-Infarct Dementia (EPMID) Study Group. European pen-toxifylline multi-infarct dementia study. Eur Neurol 1996;36:315-21.

58 Marcusson J, Rother M, Kittner B, et al. A 12-month, randomized, placebo-controlled trial of propentofylline (HWA 285 ) in patients with dementia according to DSM-III-R. Dement Geriatr Cogn Disord 1997;8:320-8.

59 Nyenhuis DL, Gorelick PB. Vascular dementia: a contemporary review of epidemiology, diagnosis, prevention, and treatment. $\mathcal{F}$ Am Geriatr Soc 1998;46:1437-48.

60 Kuller LH, Shemanski L, Manolio T, et al. Relationship between ApoE, MRI findings, and cognitive function in the cardiovascular health study. Stroke 1998;29:388-98. 